\title{
Contributions to Zoology thanks the following reviewers for their assistance over the 2013 calendar year:
}

Dirk Ahrens

Teresa Avila Pires

Yehuda Benayahu

Jose Brito Brito

Miguel Carretero

José Costa

Joel Creed

Giuseppe Donati

Camiel Doorenweerd

Hume Douglas

Danny Eibye-Jacobsen

Claudia Fichtel

Edi Gittenberger

Dick Groenenberg

Colin Groves

Juan Guayasamin

Vaclav Gvozdik
Martin Haase

Joseph Heller

Rick Hochberg

Bert Hoeksema

Slava Ivanenko

Ana Ivanović

Daniela Kalthoff

Tobias Kanneby

Philippe Kok

Bas Kokshoorn

Andrew Mackie

Benjamin Mimee

José Padial

Richard Preece

Mike Richardson

Daniel Rittschof

María del Rosario Castañeda
Kees Roselaar

Luis San-Jose

Andrew Scardino

Subbotin Sergei

Daniel Shain

Fam Shun Deng

Mark Siddall

Yann Surget-Groba

Chirasak Sutcharit

Tom van Dooren

Oscar Vorst

Philipp Wagner

Simon Webster

Ben Wielstra

Amir Yassin

Janine Ziermann

Marco Zuffi

Over the 2013 calendar year 64 manuscripts were received and 15 were published.

Publication dates were March 12 for issue 1, July 3 for issue 2, October 9 for issue 3 and December 30 for issue 4 . 